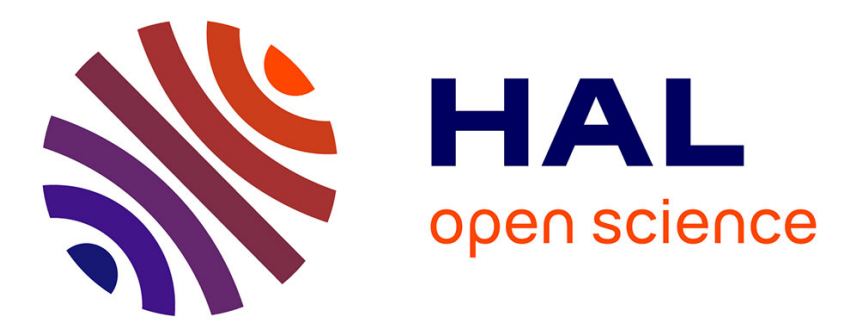

\title{
Construction of Hierarchic Families of Finite Elements by Localization of Their Degrees of Freedom
}

Cédric Doucet, Isabelle Charpentier, Jean-Louis Coulomb, Christophe Guérin

\section{To cite this version:}

Cédric Doucet, Isabelle Charpentier, Jean-Louis Coulomb, Christophe Guérin. Construction of Hierarchic Families of Finite Elements by Localization of Their Degrees of Freedom. IEEE Transactions on Magnetics, 2009, 45 (3), pp.1304-1307. 10.1109/TMAG.2009.2012605 . hal-00392798

\section{HAL Id: hal-00392798 \\ https://hal.science/hal-00392798}

Submitted on 9 Jun 2009

HAL is a multi-disciplinary open access archive for the deposit and dissemination of scientific research documents, whether they are published or not. The documents may come from teaching and research institutions in France or abroad, or from public or private research centers.
L'archive ouverte pluridisciplinaire HAL, est destinée au dépôt et à la diffusion de documents scientifiques de niveau recherche, publiés ou non, émanant des établissements d'enseignement et de recherche français ou étrangers, des laboratoires publics ou privés. 


\title{
Construction of Hierarchic Families of Finite Elements by Localization of Their Degrees of Freedom
}

\author{
Cédric Doucet $^{1,2}$, Isabelle Charpentier ${ }^{3}$, Jean-Louis Coulomb ${ }^{2}$, and Christophe Guérin ${ }^{4}$ \\ ${ }^{1}$ LJK, UMR CNRS 5224, Tour IRMA, Saint Martin d'Hères 38400, France \\ ${ }^{2}$ G2ELab, UMR CNRS 5269, ENSIEG, Saint Martin d'Hères Cedex 38402, France \\ ${ }^{3}$ LPMM, UMR CNRS 7554, 57045 Metz Cedex 01, France \\ ${ }^{4}$ Cedrat Groupe, Meylan Cedex 38246, France
}

\begin{abstract}
Hierarchic families of finite elements are widely used in higher order finite-element methods. Several hierarchic sets of basis functions for nodal, edge, and face elements are proposed by different authors in scientific literature. However, no general methodology exists for their construction. This paper presents an analysis of hierarchic families of finite elements which leads to the localization of their degrees of freedom. From this localization, we derive an algebraic approach for the construction of hierarchic families of nodal, edge, and face elements. The case of tetrahedral finite elements is discussed in detail.
\end{abstract}

Index Terms-Basis functions, degrees of freedom, hierarchic families, higher order finite-element methods.

\section{INTRODUCTION}

$\mathbf{T}$ HE use of hierarchic families in higher order finite-element methods presents several advantages. First, they reduce the computational cost of the construction of sparse linear systems of equations. Second, they also often lead to the setting of better conditioned problems [13]. Third, they allow the use of block linear solvers, such as hybrid and multilevel methods [9], [14]. Unfortunately, the construction of such families is not an easy task even if several sets of hierarchic basis functions are proposed in the scientific literature. Most of them are built from geometric properties of basis functions [15], [16] or from three-term recurrences satisfied by orthogonal polynomials [1], [13]. However, these approaches are heuristic ones. Beside, some authors proposed to localize degrees of freedom for the construction of hierarchic sets of basis functions [4]-[6], [9]. Unfortunately, they do not provide any methodology for the computation of these degrees of freedom. The work proposed in this paper is directly inspired from this strategy but we derive a simple methodology for the computation of hierarchic families of finite elements from it. The layout of this paper is the following. In Section II, we recall some basic aspects of finite elements and we explain in detail the motivations of our paper. In Section III, an abstract theory of hierarchic families of finite elements is proposed. We apply the results of our analysis to the description of hierarchic families of nodal, edge, and face finite elements on tetrahedra. Finally, we propose a symbolic computing-based approach for the automatic generation of hierarchic families of finite elements.

\section{PRELIMINARIES}

\section{A. Basic Aspects of Finite Elements}

Let $\mathbb{N}_{+}$be the set of positive integers, $\Omega$ be a bounded domain in $\mathbb{R}^{n}, n \in \mathbb{N}_{+}$, and $\mathcal{T}$ be a conforming mesh of $\Omega$. A unisolvent finite element [2] is a triple $(K, V, \Sigma)$ where $K$ is a cell in $\mathcal{T}, V$ is a vector space of real-valued functions on $K$ of

Manuscript received October 07, 2008. Current version published February 19, 2009. Corresponding author: C. Doucet (e-mail: cedric.doucet@live.fr).

Digital Object Identifier 10.1109/TMAG.2009.2012605 dimension $m<\infty$, and $\Sigma$ is a basis of $V^{*}$ (the dual space of $V)$. The duality product between $V$ and $V^{*}$ is denoted by $\langle\cdot, \cdot\rangle$. The elements of $\Sigma$ are called the degrees of freedom of the finite element (d.o.f. for short). We set

$$
\Sigma=\left\{\sigma_{1}, \ldots, \sigma_{m}\right\}
$$

This definition implies the existence of a unique basis

$$
\mathcal{B}=\left\{\phi_{1}, \ldots, \phi_{m}\right\}
$$

in $V$ such that

$$
\left\langle\sigma_{i}, \phi_{j}\right\rangle= \begin{cases}1, & \text { if } i=j \\ 0, & \text { otherwise }\end{cases}
$$

for $1 \leq i, j \leq m$. The elements of $\mathcal{B}$ are called the basis functions of $(K, \bar{V}, \Sigma)$. To each function $u$ of a functional space $F$, one can associate a unique interpolate $\Pi_{K}(u) \in V$ on $K$ so that

$$
\sigma(u)=\sigma\left(\Pi_{K}(u)\right), \quad \forall \sigma \in \Sigma .
$$

This interpolate is defined by

$$
\Pi_{K}(u)=\sum_{i=1}^{m} \sigma_{i}(u) \phi_{i}
$$

under the following assumptions:

1) $V \subset F$;

2) the definition of the d.o.f. in $\Sigma$ can be extended to $F$.

This enables defining a global interpolate $\Pi$ so that

$$
(\Pi(u))_{\mid K}=\Pi_{K}\left(u_{\mid K}\right), \quad \forall K \in \mathcal{T}
$$

for any function $u$ defined on $\Omega$. This global interpolate is said to be conforming to a functional space $H$ (or $H$-conforming for short) if, for any two adjacent cells $K_{1}, K_{2} \in \mathcal{T}$, the function defined by $\Pi_{K_{1}}\left(u_{\mid K_{1}}\right)$ on $K_{1}$ and $\Pi_{K_{2}}\left(u_{\mid K_{2}}\right)$ on $K_{2}$ belongs to $H$. We may give some precisions about the space $H$. The space of measurable functions $f: \Omega \rightarrow \mathbb{R}$ satisfying

$$
\int_{\Omega}|f(x)|^{2} \mathrm{dx}<\infty
$$

is denoted by $L^{2}(\Omega)$. For $m \in \mathbb{N}_{+}$, we use the notation $L^{2}(\Omega)^{m}$ to denote the space $\left(L^{2}(\Omega)\right)^{m}$. The use of nodal, edge, and face 
finite elements corresponds to different choices for the space $H$ and different continuity properties between adjacent mesh cells. Let $\Gamma$ be the interface between two adjacent cells $K_{1}$ and $K_{2}$ in $\mathcal{T}$. The use of nodal finite elements corresponds to the choice

$$
H=H^{1}(\Omega)=\left\{u \in L^{2}(\Omega) \mid \operatorname{grad}(u) \in L^{2}(\Omega)^{3}\right\} .
$$

A function $u \in L^{2}\left(K_{1}\right) \cup L^{2}\left(K_{2}\right)$ is in the space $H^{1}\left(K_{1} \cup\right.$ $\left.K_{2} \cup \Gamma\right)$ if and only if $u$ is the same on each side of $\Gamma$. The use of edge finite elements corresponds to the choice

$$
H=H(\operatorname{curl} ; \Omega)=\left\{u \in L^{2}(\Omega)^{3} \mid \operatorname{curl}(u) \in L^{2}(\Omega)^{3}\right\} .
$$

A function $u \in H^{1}\left(K_{1}\right)^{3} \cup H^{1}\left(K_{2}\right)^{3}$ is in the space $H\left(\operatorname{curl} ; K_{1} \cup K_{2} \cup \Gamma\right)$ if and only if the trace $u \times n$ is the same on each side of $\Gamma$ where $n$ is normal to $\Gamma$ and $\times$ denotes the cross product between vectors. Finally, the use of face finite elements corresponds to the choice

$$
H=H(\operatorname{div} ; \Omega)=\left\{u \in L^{2}(\Omega)^{3} \mid \operatorname{div}(u) \in L^{2}(\Omega)\right\} .
$$

A function $u \in H^{1}\left(K_{1}\right)^{3} \cup H^{1}\left(K_{2}\right)^{3}$ is in the space $H\left(\operatorname{div} ; K_{1} \cup K_{2} \cup \Gamma\right)$ if and only if the trace $u \cdot n$ is the same on each side of $\Gamma$ where "." denotes the Euclidean scalar product.

\section{B. Motivations of This Work}

Several families of finite elements in $H^{1}, H$ (curl) and $H$ (div) exist in the literature [10]-[12] but they are not hierarchic [7]. Several authors propose hierarchic sets of basis functions for different types of cells in various functional spaces. Some works are based on the combination of polynomial and geometrical approaches [15], [16]. Other authors build their basis functions from three-term recurrences satisfied by some orthogonal polynomials [1], [13]. Unfortunately, the genericity of nonhierarchic families is lost and these approaches are only heuristic ones. The main reason for this is that these approaches are often dedicated to projection-based interpolations [3]. In projection-based interpolation, finite elements are not defined as triples $(K, V, \Sigma)$ but as triples $\left(K, V, \Pi_{K}\right)$. In other words, the d.o.f. of hierarchic families of finite elements is not described contrary to the case of classical families [10]-[12]. In addition, corresponding basis functions do not always lead to optimally conditioned problems, and a supplementary numerical orthogonalization of some higher order basis functions is often necessary to minimize the condition number of linear systems [17]. Nevertheless, in [4]-[6], some properties of the d.o.f. of hierarchic families of finite elements are derived but no technique for their computation is proposed. This method is also used in [9]. Inspired from [4], our contribution provides a general methodology for the computation of d.o.f. of hierarchic families of finite elements. By identifying higher order d.o.f. to some scalar products, we succeed in describing hierarchic families of finite elements for the standard functional spaces $H^{1}, H$ (curl) and $H$ (div).

\section{ABSTRACT THEORY OF HIERARCHIC FAMILIES}

In this section, we propose localizing the d.o.f. of hierarchic families of finite elements for any kind of conformity. For $p \in$ $\mathbb{N}_{+}$, we consider a family of unisolvent finite elements

$$
\mathcal{F}^{(p)}=\left(\left(K, V_{k}, \Sigma_{k}\right)\right)_{1 \leq k \leq p}
$$

satisfying

$$
V_{k} \subset V_{k+1}, 1 \leq k<p .
$$

We set $m_{k}=\operatorname{dim}\left(V_{k}\right)=\operatorname{dim}\left(\Sigma_{k}\right), 1 \leq k \leq p$. The corresponding d.o.f. is denoted by

$$
\Sigma_{k}=\left\{\sigma_{1}^{(k)}, \ldots, \sigma_{m_{k}}^{(k)}\right\}, \quad 1 \leq k \leq p .
$$

The corresponding sets of basis of functions are

$$
\mathcal{B}_{k}=\left\{\phi_{1}^{(k)}, \ldots, \phi_{m_{k}}^{(k)}\right\}, \quad 1 \leq k \leq p .
$$

We assume that the family $\mathcal{F}^{(p)}$ is hierarchic, that is, to say it satisfies

$$
\mathcal{B}_{k} \subset \mathcal{B}_{k+1}, \quad 1 \leq k<p .
$$

In other words, first elements in $\mathcal{B}_{k+1}$ are the elements of $\mathcal{B}_{k}$ since

$$
\phi_{j}^{(k+1)}=\phi_{j}^{(k)}, \quad 1 \leq k<p
$$

for $j=1, \ldots, m_{k}$. We assume that a canonical basis $\left\{e_{1}, \ldots, e_{m_{p}}\right\}$ of $V_{p}$ is known. From (12), it is clear that

$$
V_{k}=\operatorname{span}\left\{e_{1}, \ldots, e_{m_{k}}\right\}, \quad 1 \leq k \leq p
$$

and any basis function $\phi_{j}^{(k)} \in \mathcal{B}_{k}$ can be uniquely written in the form

$$
\phi_{j}^{(k)}=\sum_{l=1}^{m_{k}} a_{l j} e_{l} .
$$

Let $k<p$. From (3) and (18), we obtain

$$
\left\langle\sigma_{i}^{(k+1)}, e_{l}\right\rangle=\left\langle\sigma_{i}^{(k)}, e_{l}\right\rangle, \quad l=1, \ldots, m_{k}
$$

for $1 \leq i \leq m_{k}$. This is equivalent to

$$
\forall p \in V_{k},\left\langle\sigma_{i}^{(k+1)}, p\right\rangle=\left\langle\sigma_{i}^{(k)}, p\right\rangle
$$

for $1 \leq i \leq m_{k}$. This implies that the first $m_{k}$ d.o.f. of $\Sigma_{k+1}$ are the respective extensions to $V_{k+1}$ of the $m_{k}$ d.o.f. in $\Sigma_{k}$. Therefore, we only need to describe the last $m_{k+1}-m_{k}$ d.o.f. in $\Sigma_{k+1}$. From (3) and (15), we obtain

$$
\left\langle\sigma_{i}^{(k+1)}, \phi_{j}^{(k)}\right\rangle=0
$$

for $i=m_{k}+1, \ldots, m_{k+1}$ and $j=1, \ldots, m_{k}$. Since $\mathcal{B}_{k}$ is a basis of $V_{k},(21)$ is equivalent to

$$
\forall p \in V_{k}, \quad\left\langle\sigma_{i}^{(k+1)}, p\right\rangle=0
$$

$i=m_{k}+1, \ldots, m_{k+1}$. Thus, if a family $\mathcal{F}^{(p)}$ of unisolvent finite elements is hierarchic, conditions (20) and (22) are necessarily satisfied. Conversely, it can be proved that conditions (20) and (22) are sufficient for the hierarchy of $\mathcal{F}^{(p)}$. Condition (22) was already mentioned and used for the construction of hierarchic families in [4]-[6], [9]. However, these authors do not mention how to choose d.o.f. and they do not provide any general definition of hierarchic families of finite elements in the spaces $H^{1}, H($ curl), and $H($ div $)$. In the next section, we give a general description of each family by identifying the d.o.f. of classical families of finite element to scalar products. 


\section{IMPOSING HIERARCHY TO EXISTING FAMILIES}

For any open subset $\Omega \subset \mathbb{R}^{n}, n \in \mathbb{N}_{+}$, the scalar product in $L^{2}(\Omega)^{m}, m \in \mathbb{N}_{+}$, is defined by

$$
(f, g)_{L^{2}(\Omega)^{m}}=\int_{\Omega} f(x) \cdot g(x) \mathrm{dx}, \quad \forall f, g \in L^{2}(\Omega)^{m} .
$$

In the next subsection, we describe classical families of finite elements by identifying their d.o.f. to such scalar products. They exactly correspond to those described in [10]-[12]. We introduce some notations for this task. Let $K$ be a mesh cell. The d.o.f. of finite elements may be associated with each vertex $v$, each edge $e$, each face $f$ of $K$, or $K$ itself. The length of an edge $e$ is denoted by $\int_{e} d l$ and the unit vector along $e$ is denoted by $\tau_{e}$. The area of a face $f$ is denoted by $\int_{f} d s$, and $n_{f}$ is the unit outward normal vector on a face $f$. Finally, the volume of $K$ is denoted by $\int_{K} \mathrm{dx}$.

\section{A. Classical Families on Tetrahedra}

Families of finite elements in the spaces $H^{1}, H$ (curl), and $H$ (div) can be described in a generic fashion. We only consider some finite elements on tetrahedra but similar considerations can be made for the families proposed in [10]-[12]. Let $K=$ $\mathcal{K}_{T}$ be a tetrahedron in the mesh $\mathcal{T}$ and $\mathcal{E}_{k}=\left(\mathcal{K}_{T}, V_{k}, \Sigma_{k}\right)$, $1<k \leq p$, be a unisolvent finite element belonging to a family $\mathcal{F}_{p}$. The choice of the space $V_{k}$ depends on the choice of the space $H$. Following [10] and [11], we define several spaces of polynomials. The space of polynomials of a degree less than or equal to $k$ is denoted by $P_{k}$, and $\tilde{P}_{k}$ is the space of homogenenous polynomials of a degree equal to $k$. We set

$$
D_{k}=\left(P_{k-1}\right)^{3} \oplus \tilde{P}_{k-1} \cdot x
$$

and

$$
R_{k}=\left(P_{k-1}\right)^{3} \oplus S_{k}
$$

where

$$
S_{k}=\left\{p \in\left(\tilde{P}_{k}\right)^{3} \mid \forall x \in \mathbb{R}^{3}, p(x) \cdot x=0\right\} .
$$

Let $p \in V_{k}$. The d.o.f. of classical families in the spaces $H^{1}$, $H$ (curl), and $H$ (div) will be described. They involve some polynomial spaces which are given in Table I. If $\mathcal{E}_{k}$ is $H^{1}$-conforming, then $V_{k}=P_{k}$ and the d.o.f. in $\Sigma_{k}$ can be expressed in the following form:

$$
\begin{aligned}
& \left\langle\sigma_{v}^{(1)}, p\right\rangle=p(v) \\
& \left\langle\sigma_{e}^{(k)}, p\right\rangle=(p, q)_{L^{2}(e)}, q \in \mathcal{N}_{k}^{e} \\
& \left\langle\sigma_{f}^{(k)}, p\right\rangle=(p, q)_{L^{2}(f)}, q \in \mathcal{N}_{k}^{f} \\
& \left\langle\sigma_{K}^{(k)}, p\right\rangle=(p, q)_{L^{2}(K)}, q \in \mathcal{N}_{k}^{K} .
\end{aligned}
$$

If $\mathcal{E}_{k}$ is $H$ (curl)-conforming, then $V_{k}=R_{k}$ and the corresponding d.o.f. are

$$
\begin{aligned}
& \left\langle\sigma_{e}^{(k)}, p\right\rangle=\left(p \cdot \tau_{e}, q\right)_{L^{2}(e)}, q \in \mathcal{E}_{k}^{e} \\
& \left\langle\sigma_{f}^{(k)}, p\right\rangle=\left(p \times n_{f}, q\right)_{L^{2}(f)^{2}}, q \in \mathcal{E}_{k}^{f} \\
& \left\langle\sigma_{K}^{(k)}, p\right\rangle=(p, q)_{L^{2}(K)^{3}}, q \in \mathcal{E}_{k}^{K} .
\end{aligned}
$$

TABLE I

Classical AND NEW Higher ORDER d.o.f. FOR TETRAHEDRA

\begin{tabular}{|c|c|c|c|}
\hline & Classical & New & Order \\
\hline $\mathcal{N}_{k}^{e}$ & $P_{k-2}(e)$ & $\left(P_{k-1}(e)\right)^{\perp} \cap P_{k}(e)$ & $k>1$ \\
\hline $\mathcal{N}_{k}^{f}$ & $P_{k-3}(f)$ & $\left(P_{k-1}(f)\right)^{\perp} \cap P_{k}(f)$ & $k>2$ \\
\hline $\mathcal{N}_{k}^{K}$ & $P_{k-4}(K)$ & $\left(P_{k-1}(K)\right)^{\perp} \cap P_{k}(K)$ & $k>3$ \\
\hline $\mathcal{E}_{k}^{e}$ & $P_{k-1}(e)$ & $\left(P_{k-1}(e)\right)^{\perp} \cap P_{k}(e)$ & $k>1$ \\
\hline $\mathcal{E}_{k}^{f}$ & $\left(P_{k-2}(f)\right)^{2}$ & $\begin{array}{c}\left(\left(P_{k-1}(f)\right)^{2}\right)^{\perp} \cap \\
\left(P_{k}(f)\right)^{2}\end{array}$ & $k>2$ \\
\hline $\mathcal{E}_{k}{ }^{K}$ & $\left(P_{k-3}(K)\right)^{3}$ & $\begin{array}{c}\left(\left(P_{k-1}(K)\right)^{3}\right)^{\perp} \cap \\
P_{k}(K)^{3}\end{array}$ & $k>3$ \\
\hline$F_{k}^{f}$ & $P_{k-1}(f)$ & $\left(P_{k-1}(f)\right)^{\perp} \cap P_{k}(f)$ & $k>1$ \\
\hline$F_{k}{ }^{K}$ & $\left(P_{k-2}(K)\right)^{3}$ & $\begin{array}{c}\left(\left(P_{k-1}(K)\right)^{3}\right)^{\perp} \cap \\
\left(P_{k}(K)\right)^{3}\end{array}$ & $k>2$ \\
\hline
\end{tabular}

Finally, if $\mathcal{E}_{k}$ is $H$ (div)-conforming, $V_{k}=D_{k}$ and its d.o.f. are

$$
\begin{aligned}
& \left\langle\sigma_{f}^{(k)}, p\right\rangle=\left(p \cdot n_{f}, q\right)_{L^{2}(f)}, q \in \mathcal{F}_{k}^{f} \\
& \left\langle\sigma_{K}^{(k)}, p\right\rangle=(p, q)_{L^{2}(K)^{3}}, q \in \mathcal{F}_{k}^{K} .
\end{aligned}
$$

It is well known that these classical families are not hierarchic. For example, the first higher order basis functions described by the d.o.f. (31)-(33) are given explicitly in [7]. In the next subsection, we explain how to impose hierarchy to these families.

\section{B. Hierarchic Families on Tetrahedra}

The idea behind our approach is to modify the definition of the classical d.o.f. in order to impose (21). Since we identified these d.o.f. with scalar products in the space $L^{2}$, we proceed by orthogonalization to form new d.o.f. First, we consider nodal finite elements. For $k=1$, the d.o.f. in (27) are the same. For $k>1$ and any subset $U$ of $\mathbb{R}^{m}$, we define $\left(\left(P_{k-1}(U)\right)^{m}\right)^{\perp}$ as the orthogonal space of $\left(P_{k-1}(U)\right)^{m}$ in $L_{2}(U)^{m}$

$$
\left\{q \in L_{2}(U)^{m} \mid \forall p \in\left(P_{k-1}(U)\right)^{m},(p, q)_{L^{2}(U)^{m}}=0\right\} .
$$

Let $p \in P_{k}$. The restriction of $p$ on any edge $e$ (respectively, face $f$ ) belongs to $P_{k}(e)$ (respectively, $P_{k}(f)$ ). Thus, choosing $\mathcal{N}_{k}^{e}=\left(P_{k-1}(e)\right)^{\perp} \cap P_{k}(e), \mathcal{N}_{k}^{f}=\left(P_{k-1}(f)\right)^{\perp} \cap P_{k}(f)$, and $\mathcal{N}_{k}^{K}=P_{k-1}(K)^{\perp} \cap P_{k}(K)$, we satisfy (3), (20), and (22) at any order $k>1$ : the resulting family is unisolvent, hierarchic and $H^{1}$-conforming on $\mathcal{K}_{T}$. The generalization of this orthogonalization process in the spaces $H$ (curl) and $H$ (div) is obtained by following a similar approach. For example, let us consider the case of finite elements in the space $H$ (curl) and let $p \in R_{k}$. Of course, the first-order d.o.f. remain unchanged. The restriction of $p \cdot \tau_{e}$ (respectively, $p \times n_{f}$ ) on any edge $e$ (respectively, face $f$ ) belongs to $P_{k}(e)$ (respectively, $\left.\left(P_{k}(f)\right)^{2}\right)$. Thus, we choose $\mathcal{E}_{k}^{e}=\left(P_{k-1}(e)\right)^{\perp} \cap P_{k}(e)$ and $\mathcal{E}_{k}^{f}=\left(\left(P_{k-1}(f)\right)^{2}\right)^{\perp} \cap\left(P_{k}(f)\right)^{2}$, etc. The complete description of our new d.o.f. is given in Table I. The construction process for other mesh cells is exacly the same, except that the definition of the space $V_{k}$ is different.

\section{Symbolic CONSTRUCTION}

One difficulty in programming the finite-element method is the complexity of generating higher order basis functions. In this section, we explain how to generate them automatically. The idea is to use the property of hierarchy to allow the symbolic 
computing of d.o.f.: it requires the implementation of an orthogonalization process. Then, the symbolic computing of basis functions is straightforward considering (3) and only requires the implementation of a dense direct linear solver.

\section{A. Degrees of Freedom}

The d.o.f. of hierarchic families of finite elements depend on a polynomial $q$, which belongs to the orthogonal of a polynomial space in a $L^{2}$-type scalar product. Thus, computing d.o.f. is equivalent to computing a polynomial $q$ in an orthogonal space. For example, let $\left\{p_{1}, \ldots, p_{l}\right\}$ be a set of linearly independent polynomials in $P_{k}(U)^{m}, l>1$. An orthogonal basis $\left\{q_{1}, \ldots, q_{l}\right\}$ of $P_{k}(U)^{m}$ may be computed according to the scalar product $(\cdot, \cdot)_{L^{2}(U)^{m}}$ by applying the methods of Gram-Schmidt or Householder to the set $\left\{p_{1}, \ldots, p_{l}\right\}$ [8]. Assuming $\left\{p_{1}, \ldots, p_{j}\right\}$ spans $P_{k-1}(U)^{m} \subset P_{k}(U)^{m}$ and $\left\{p_{j+1}, \ldots, p_{l}\right\}$ spans $\widetilde{P}_{k}(U)^{m}$ for some $j<l$, it follows that $\left\{q_{j+1}, \ldots, q_{l}\right\}$ forms a basis of $\left(P_{k-1}(U)^{m}\right)^{\perp} \cap P_{k}(U)^{m}$. Thus, $q$ may be computed as a nontrivial linear combination of the elements $q_{i}, i=j+1, \ldots, l$. Let us remark that families of orthogonal polynomials may also be used directly if some are known.

\section{B. Basis Functions}

We use the notations introduced in Section III. Any basis function $\phi_{j}^{(k+1)} \in V_{k+1}, m_{k}+1 \leq j \leq m_{k+1}$, can be uniquely written in the form

$$
\phi_{j}^{(k+1)}=\sum_{l=1}^{m_{k}} a_{j l} e_{l}+\sum_{l=m_{k}+1}^{m_{k+1}} a_{j l} e_{l}=\varphi_{j}^{(k+1)}+\psi_{j}^{(k+1)}
$$

where $\varphi_{j}^{(k+1)} \in V_{k}$ and $\psi_{j}^{(k+1)} \in V_{k+1} \backslash V_{k}$. Considering (3), we obtain that the coefficients appearing in functions $\psi_{j}^{(k+1)}$ are the unique solution of the block linear system of equations

$$
S_{1} A_{1}=I,
$$

where

$$
S_{1}(i, l)=\left\langle\sigma_{i}^{(k+1)}, e_{l}\right\rangle
$$

for $m_{k}+1 \leq i, l \leq m_{k+1}, A_{1}(j, l)=a_{j l}$ for $m_{k}+1 \leq j, l \leq$ $m_{k+1}$ and $I$ is the identity matrix. Then, coefficients appearing in the functions $\varphi_{j}^{(k+1)}$ are computed by solving another block linear system of equations

$$
S_{2} A_{2}=-B
$$

where

$$
S_{2}(i, l)=\left(\left\langle\sigma_{i}^{(k)}, e_{l}\right\rangle\right)
$$

for $1 \leq i, l \leq m_{k}, A_{2}(l, j)=a_{l j}$ for $1 \leq l \leq m_{k}$ and $m_{k}+1 \leq j \leq m_{k+1}$. The block right-hand side is defined by $B(i, j)=<\sigma_{i}^{(k+1)}, \psi_{j}^{(k+1)}>$ for $1 \leq i \leq m_{k}$ and $m_{k}+1 \leq$ $j \leq m_{k+1}$. In practice, basis functions are computed for a small order $p$. Then, they may be implemented in a finite-element code by copying their respective expressions in a subprogram.

\section{CONCLUSION}

In this paper, we describe hierarchic families of finite elements in a very general framework. Our analysis shows that hierarchy is obtained from a judicious localization of the d.o.f. of classical families of finite elements. As a consequence, the construction of hierarchic families in the spaces $H^{1}, H$ (curl), and $H$ (div) is simply performed by modifying the d.o.f. of the corresponding nonhierarchic families previously proposed. The localization of d.o.f. proposed in this paper leads to the setting of a symbolic process for the explicit construction of higher order basis functions. This process requires the implementation of an orthogonalization method and a linear system solution only. The characterization of d.o.f. satisfying additional properties (optimal conditioning, exact sequence property, etc.) is under study.

\section{REFERENCES}

[1] M. Ainsworth and J. Coyle, "Hierarchic finite element bases on unstructured tetrahedral meshes," Int. J. Numerical Methods Eng., vol. 58, no. 14, pp. 2103-2130, 2003.

[2] P. G. Ciarlet, "The finite element method for elliptic problems," in Studies in Mathematics and its Applications. Amsterdam, The Netherlands: North-Holland, 1978.

[3] L. Demkowicz, "Projection based interpolation," ICES Rep. 04-03, 2004.

[4] C. Geuzaine, "Développement d'éléments finis nodaux et d'arête hiérarchiques $2 \mathrm{D}$ et 3D appliqués au problème des courants induits," Graduate thesis, Univ. Liège, Liège, Belgium, May 1996, Fac. Appl. Sci.

[5] C. Geuzaine, "High order hybrid finite element schemes for Maxwell's equations taking thin structures and global quantities into account," Ph.D. dissertation, Univ. Liège, Liège, Belgium, Dec. 2001, Fac. Appl. Sci.

[6] C. Geuzaine, B. Meys, P. Dular, and W. Legros, "Convergence of higher-order curl-conforming finite elements," IEEE Trans. Magn., vol. 35 , no. 3 , pt. 1 , pp. $1442-1445$, May 1999.

[7] J. Gopalakrishnan, L. E. García-Castillo, and L. F. Demkowicz, "Nédélec spaces in affine coordinates," Comput. Math. Appl., vol. 49, no. 7-8, pp. 1285-1294, 2005.

[8] A. S. Householder, The Theory of Matrices in Numerical Analysis. Johnson, CO: Blaisedell, 1964.

[9] P. Ingelstöm, "A new set of H(curl)-conforming hierarchical basis functions for tetrahedral meshes," IEEE Trans. Microw. Theory Tech., vol. 54, no. 1, pp. 106-114, Jan. 2006.

[10] P. Monk, Finite Element Methods for Maxwell's Equations. Londo, U.K.: Oxford Science .

[11] J.-C. Nédélec, "Mixed finite elements in $\mathbb{R}^{3}, "$ Numer. Math., vol. 35, pp. 315-341, 1980.

[12] J.-C. Nédélec, "A new family of mixed finite elements in $\mathbb{R}^{3}$," Numer Math., vol. 50, pp. 57-81, 1986.

[13] P. Solin, K. Segeth, and I. Dolezel, Higher-Order Finite Element Methods. Boca Raton, FL: CRC, 2003.

[14] J. P. Webb, "Combined direct-iterative matrix solvers for hierarchal vector finite elements," IEEE Trans. Magn., vol. 38, no. 2, pt. 1, pp. 345-348, Mar. 2002.

[15] J. P. Webb, "Hierarchal vector basis functions of arbitrary order for triangular and tetrahedral finite elements," IEEE Trans. Antennas Propag., vol. 47, no. 8, pp. 1244-1253, Aug. 1999.

[16] J. P. Webb and B. Forghani, "Hierarchal scalar and vector tetrahedra," IEEE Trans. Magn., vol. 29, no. 2, pp. 1495-1498, Mar. 1993.

[17] M. Zitka, P. Solin, T. Vejchodsky, and F. Avila, "Imposing orthogonality to hierarchic higher-order finite elements," Math. Comp. Sim., 2007. 